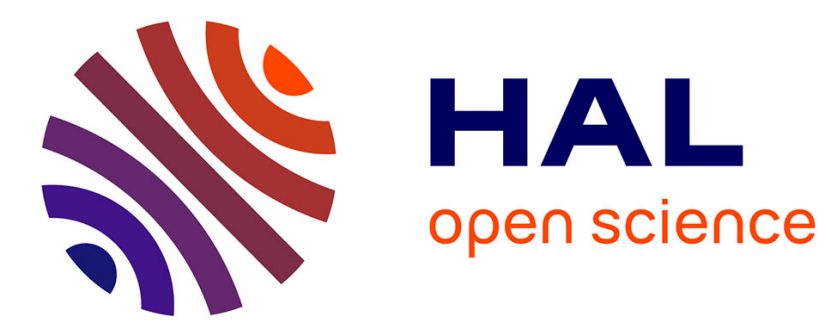

\title{
La sensibilité de la détection de la R. P. E. en utilisant l'effet Faraday
}

\author{
E. Tătaru
}

\section{To cite this version:}

E. Tătaru. La sensibilité de la détection de la R. P. E. en utilisant l'effet Faraday. Revue de Physique Appliquée, 1974, 9 (3), pp.521-524. 10.1051/rphysap:0197400903052100 . jpa-00243812

\section{HAL Id: jpa-00243812 https://hal.science/jpa-00243812}

Submitted on 1 Jan 1974

HAL is a multi-disciplinary open access archive for the deposit and dissemination of scientific research documents, whether they are published or not. The documents may come from teaching and research institutions in France or abroad, or from public or private research centers.
L'archive ouverte pluridisciplinaire HAL, est destinée au dépôt et à la diffusion de documents scientifiques de niveau recherche, publiés ou non, émanant des établissements d'enseignement et de recherche français ou étrangers, des laboratoires publics ou privés. 


\title{
LA SENSIBILITÉ DE LA DÉTECTION DE LA R. P. E. EN UTILISANT L'EFFET FARADAY
}

\author{
E. TǍTARU \\ Faculté de Physique, Université Cluj, Str. Kogălniceanu Nr. 1 Cluj, Roumanie
}

(Reçu le 6 novembre 1973, révisé le 15 janvier 1974)

\begin{abstract}
Résumé. - Dans ce travail, on détermine le rapport signal-bruit, le nombre minimum de spins détectables, les conditions de travail optimum et les particularités de la détection de la R.P.E. en utilisant la rotation Faraday ou le dichroïsme circulaire magnétique.
\end{abstract}

\begin{abstract}
This paper discusses the signal to noise ratio, the minimum detectable number of spins, the optimum experimental conditions, and certain peculiarities of electron-paramagneticresonance detection by means of either the Faraday effect or of magnetic circular dichroism.
\end{abstract}

1. Introduction. - En 1951, le professeur A. Kastler [1] a montré que la résonance magnétique électronique des ions paramagnétiques doit influencer la polarisation rotatoire de ces ions, c'est-à-dire que la résonance paramagnétique électronique (R. P. E.) peut être détectée à l'aide de l'effet Faraday observé dans le domaine des radiations visibles. W. Opechowski (1953) [2] a donné un traitement quantique de l'influence de la R. P. E. sur la rotation Faraday et J. M. Daniels et H. Wesemeyer (1958) [3] ont mis en œuvre expérimentalement l'idée du prof. A. Kastler. C. K. Asawa et R. A. Satten (1962) [4] ont détecté la R. P. E. en repérant la variation provoquée sur la lumière circulairement polarisée transmise par l'échantillon. L. F. Mollenauer et al. (1969) [5] ont utilisé le dichroïsme circulaire magnétique (D. C. M.) pour la détection de la R. P. E.

Dans le présent article nous nous proposons de déterminer le rapport signal-bruit, la sensibilité (le nombre minimal de spins détectable), les conditions optimales de travail et de relever les particularités de la détection de la R. P. E. de l'état fondamental en utilisant l'effet Faraday. Il faut signaler que S. Geschwind [6] traite brièvement le problème du rapport signal-bruit pour la détection optique de la R. P. E. d'un état excité, problème voisin mais distinct de celui examiné ci-dessous.

2. Théorie. - Comme on sait déjà, l'effet Faraday implique deux aspects : la rotation Faraday et le dichroïsme circulaire magnétique [7]. La composante paramagnétique de l'effet Faraday est proportionnelle à la magnétisation et, comme les transitions R. P. E. font varier la magnétisation, nous avons une variation de l'effet Faraday qui peut être utilisée pour l'enregis- trement de la R. P. E. En conséquence, on peut faire l'enregistrement de la R. P. E. des manières suivantes :

1) On enregistre la rotation Faraday ou le D. C. M. en fonction du champ magnétique en présence du champ magnétique de microondes. Dans ce cas, le signal R. P. E. paraît sur le fond de l'effet Faraday $[3,5]$. Ce fond peut être éliminé en faisant la différence avec la courbe d'effet Faraday enregistrée en l'absence du champ magnétique de microondes.

2) On module en impulsions rectangulaires le champ de microondes et on enregistre la variation de l'effet Faraday causée par les transitions R. P. E. La période du signal modulateur doit être plus grande que le temps de relaxation longitudinale en vue d'obtenir le maximum d'efficacité de la modulation [8]. Comme on voit aisément, la deuxième méthode a l'avantage d'enregistrer directement le signal R. P. E., le fond perturbateur étant absent.

Dans les deux cas la longueur d'onde du faisceau lumineux est choisie afin que l'effet Faraday soit maximum.

Pour déterminer l'influence de la R. P. E. sur l'effet Faraday nous partons de la relation établie dans [9] pour le cas d'un doublet de Kramers :

$$
\varepsilon=2 l_{0}(\lambda) \Gamma(\lambda) P
$$

où $\varepsilon$ est le dichroïsme circulaire magnétique, $l$ la longueur du chemin optique dans l'échantillon à étudier, $\gamma_{0}(\lambda)$ le coefficient d'absorption en lumière non polarisée et en champ magnétique nul, $\Gamma(\lambda)$ le coefficient de D. C. M., $\lambda$ la longueur d'onde,

$$
P=\left(\mathrm{n}_{+}-\mathrm{n}_{-}\right) /\left(\mathrm{n}_{+}+\mathrm{n}_{-}\right)
$$

la polarisation électronique de l'état fondamental, 
$\mathbf{n}_{+}$et $\mathbf{n}_{-}$les populations des deux sous-niveaux. L'utilisation de la formule (1) suppose [9] que

$$
|\varepsilon| \ll 1 \text { et }\left|l\left[\gamma_{+}(\lambda)-\gamma_{-}(\lambda)\right]\right| \ll 1
$$

où $\gamma_{ \pm}(\lambda)$ représente les coefficients d'absorption en lumière circulairement polarisée. Ces conditions sont justifiées ici puisqu'on s'intéresse à la sensibilité ultime de la détection optique, donc à des échantillons contenant très peu de centres paramagnétiques du type étudié.

Dans le but de déterminer le nombre minimal détectable de spins célibataires, il est convenable d'écrire (1) sous une autre forme, en tenant compte de ce que :

$$
\gamma_{0} l=\sigma_{\mathrm{a}} N_{0} l=\sigma_{\mathrm{a}}\left(\frac{N}{A}\right)
$$

où $\sigma_{\mathrm{a}}$ est la section efficace d'absorption, $N_{0}$ la concentration par unité de volume des ions paramagnétiques, $N$ le nombre total des ions paramagnétiques qui se trouvent sous l'action du faisceau lumineux et $A$ l'aire illuminée de la section transversale de l'échantillon. De (1) et (2), il résulte :

$$
\varepsilon=2 \sigma_{\mathrm{a}} \Gamma P\left(\frac{N}{A}\right)=\sigma_{\varepsilon}\left(\frac{N}{A}\right)
$$

où nous avons introduit la grandeur $\sigma_{\varepsilon}=2 \sigma_{\mathrm{a}} \Gamma P$ qui, par analogie, est nommée section transversale efficace du dichroïsme circulaire magnétique. En tenant compte de ce que le D. C. M. $(\varepsilon)$ et la rotation Faraday $(\varphi)$ sont liés par les relations de Kramers-Krönig [10] on peut écrire :

$$
\varphi=\sigma_{\varphi}\left(\frac{N}{A}\right)
$$

où la grandeur $\sigma_{\varphi}$ représente la section transversale efficace qui correspond à la rotation Faraday. Parce que $\sigma_{\varepsilon}$ est proportionnelle à la polarisation électronique $P$, qui est indépendante de $\lambda$, et que $\sigma_{\varepsilon}$ et $\sigma_{\varphi}$ sont liées par les relations de Kramers-Krönig, il résulte que $\sigma_{\varphi}$ est aussi proportionnelle à $P$.

La détection de la R. P. E. en utilisant l'effet Faraday se base sur le fait que les transitions R. P. E. provoquent la variation de la différence des populations $\left(\mathrm{n}_{+}-\mathrm{n}_{-}\right)$, c'est-à-dire une variation de la polarisation électronique, ce qui cause une variation de l'effet Faraday. En particulier, à saturation de la transition R. E. P. nous avons $\left(n_{+}-n_{-}\right)=0$, fait qui conduit à l'annulation de la composante paramagnétique de l'effet Faraday. Un calcul simple de la variation $\delta\left(n_{+}-n_{-}\right)$de la différence des populations causée par les transitions R. P. E., dans le cas d'un système de $\operatorname{spin} S=\frac{1}{2}$ et d'une raie de Lorentz, nous donne

$$
\delta\left(\mathrm{n}_{+}-\mathrm{n}_{-}\right)=-\frac{\left(\mathrm{n}_{+}-\mathrm{n}_{-}\right) s}{1+s+\left(T_{2} \Delta \omega\right)^{2}}
$$

où $\left(n_{+}-n_{-}\right)$est la différence des populations en l'absence de R. P. E., $T_{2}$ le temps de la relaxation transversale, $\Delta \omega=\omega-\omega_{0}, \omega_{0}$ la pulsation R. P. E., $s=\gamma^{2} H_{1}^{2} T_{1} T_{2} / 4$ le facteur de saturation, $\gamma$ le rapport gyromagnétique, $H_{1}$ l'amplitude du champ magnétique de microondes linéairement polarisé et $T_{1}$ le temps de relaxation longitudinale. Les variations relatives causées par la $R$. P. E. satisfont les relations :

$$
\frac{\delta\left(\mathrm{n}_{+}-\mathrm{n}_{-}\right)}{\left(\mathrm{n}_{+}-\mathrm{n}_{-}\right)}=\frac{\delta P}{P}=\frac{\delta \varepsilon}{\varepsilon}=\frac{\delta \varphi}{\varphi}
$$

et ainsi on peut écrire :

$$
\frac{\delta \varepsilon}{\varepsilon}=\frac{\delta \varphi}{\varphi}=-\frac{s}{1+s+\left(T_{2} \Delta \omega\right)^{2}}
$$

La dernière relation montre que les variations $\delta \varepsilon$ et $\delta \varphi$ de l'effet Faraday suivent la courbe d'absorption R. P. E.

Considérons le cas de l'enregistrement de la R. P. E. en utilisant la rotation Faraday et la modulation en impulsions rectangulaires du champ de microondes. Soit $I$ l'intensité de la lumière incidente sur l'échantillon, $\theta$ l'angle complémentaire de celui formé par les axes de transmission des polariseurs, $\Delta I$ l'intensité de la lumière reçue par le photomultiplicateur en absence de la R. P. E. et $\alpha$ le .coefficient qui caractérise les imperfections du montage. Supposons que $\gamma_{0} l \ll 1$, c'est-à-dire que l'échantillon absorbe peu de lumière. On peut écrire :

$$
\Delta I=I\left[\alpha+\sin ^{2}(\theta+\varphi)\right] .
$$

En différentiant la relation (7), nous obtenons la variation de l'intensité de la lumière causée par les transitions R. P. E. :

$$
\delta I=I \sin 2(\theta+\varphi) \delta \varphi .
$$

Soit $i_{\mathrm{c}}=\eta \Delta I$ le courant cathodique, $\eta$ la sensibilité de la photocathode, $i_{\mathrm{a}}$ le courant anodique et $G=\left(i_{\mathrm{a}} / i_{\mathrm{c}}\right)$ le gain du photomultiplicateur. Tenant compte du fait que la valeur efficace des signaux rectangulaires de lumière est $(\delta I / 2)$, nous pouvons écrire la valeur efficace de la variation du courant anodique, $\delta i_{\mathrm{a} \text { eff }}$, causée par la modulation en impulsions du champ de microondes, sous la forme :

$$
\delta i_{\mathrm{a} \text { eff }}=\frac{1}{2} G \eta I \sin 2(\theta+\varphi) \delta \varphi
$$

D'autre part, en ce qui concerne le bruit des photomultiplicateurs, le rôle principal est joué par les fluctuations Schottky de l'émission photoélectronique [11, 12]. Si nous notons $\left(i_{f \mathrm{a}}^{2 \sqrt{1 / 2}}\right.$ la valeur efficace des fluctuations du courant anodique, $q$ la charge de l'électron et $\Delta f$ la bande passante du système d'enregistrement, on peut écrire :

$$
\left(\overline{i_{f a}^{2}}\right)^{1 / 2}=G(2 q \eta \Delta I \Delta f)^{1 / 2 \coprod}
$$

De (8) et (9), en tenant compte de (4), (6) et (7), dans 
le cas de la résonance $(\Delta \omega=0)$, nous obtenons le rapport signal-bruit sous la forme :

$\frac{\delta i_{\mathrm{a} \text { eff }}}{\left.\overline{\left(i_{f \mathrm{a}}^{2}\right.}\right)^{1 / 2}}=F(\theta+\varphi) \cdot \sigma_{\varphi}\left(\frac{N}{A}\right)\left(\frac{s}{1+s}\right)\left(\frac{\eta I}{2 q \Delta f}\right)^{1 / 2}$

où

$$
F(\theta+\varphi)=\frac{\sin 2(\theta+\varphi)}{2\left[\alpha+\sin ^{2} \cdot(\theta+\varphi)\right]^{1 / 2}} .
$$

La fonction $F(\theta+\varphi)$ présente un maximum pour $(\theta+\varphi)_{\mathrm{m}}=\frac{1}{2} \operatorname{arc} \cos [1+2 \alpha-2 \sqrt{\alpha(1+\alpha)}]$.

En tenant compte de ce que dans la pratique

$$
\alpha=10^{-3} \div 10^{-5} \text {, }
$$

on peut écrire avec une bonne approximation

$$
\begin{aligned}
& \cos 2(\theta+\varphi)_{\mathrm{m}} \simeq 1-2 \sqrt{\alpha} ; \\
& \sin (\theta+\varphi)_{\mathrm{m}} \simeq(\theta+\varphi)_{\mathrm{m}} \simeq \alpha^{1 / 4} .
\end{aligned}
$$

En conséquence la valeur maximum de la fonction $F(\theta+\varphi)$ est pratiquement indépendante de $\alpha$ et a la valeur

$$
F\left[(\theta+\varphi)_{\mathrm{m}}\right]=1 .
$$

On peut observer que, aussi bien pour $(\theta+\varphi)=\pi / 4$ que pour $(\theta+\varphi)=\alpha^{1 / 2}$ la fonction $F(\theta+\varphi)$ n'est diminuée que de $\sqrt{2}$ fois par rapport à sa valeur maximum, c'est-à-dire que l'angle $(\theta+\varphi)$ n'est pas très critique. En combinant les relations (10) et (13), il résulte :

$$
\frac{\delta i_{\mathrm{a} e f f}}{\left(\overline{i_{f a}^{2}}\right)^{1 / 2}}=\sigma_{\varphi}\left(\frac{N}{A}\right)\left(\frac{s}{1+s}\right)\left(\frac{\eta I}{2 q \Delta f}\right)^{1 / 2} .
$$

D'après la relation (14), en prenant le rapport signal-bruit égal à l'unité, on obtient le nombre minimum de spins détectables

$$
N_{\min }=\left(\frac{A}{\sigma_{\varphi}}\right)\left(\frac{1+s}{s}\right)\left(\frac{2 q \Delta f}{\eta I}\right)^{1 / 2} .
$$

Dans le cas de la détection de la R. P. E. en utilisant le D. C. M. et la modulation en impulsions rectangulaires du champ de microondes, en tenant compte de ce que $i_{\mathrm{c}}=\eta I$ et $\delta I=I \delta \varepsilon$, on obtient, par des calculs analogues à ceux des équations (7) à (15), les expressions suivantes du rapport signal-bruit et du nombre minimum de spins détectables :

$$
\begin{aligned}
\frac{\delta i_{\mathrm{a} e f f}}{\left.\overline{\left(i_{f \mathrm{a}}^{2}\right.}\right)^{1 / 2}} & =\sigma_{\varepsilon}\left(\frac{N}{2 A}\right)\left(\frac{s}{1+s}\right)\left(\frac{\eta I}{2 q \Delta f}\right)^{1 / 2} \\
N_{\min } & =\left(\frac{2 A}{\sigma_{\varepsilon}}\right)\left(\frac{1+s}{s}\right)\left(\frac{2 q \Delta f}{\eta I}\right)^{1 / 2} .
\end{aligned}
$$

En ce qui concerne la détection de la R. P. E. en utilisant l'enregistrement de la rotation Faraday par la méthode Billardon [13] et la détection de la R. P. E. par la méthode Asawa-Satten [4], nous obtenons pour $N_{\min }$ le même ordre de grandeur que celui qui résulte de (15) ou de (17).
3. Discussion et conclusion. - 3.1 Des relations (14) et (16) il résulte que le rapport signal-bruit est proportionnel au nombre de spins par unité d'aire illuminée, c'est-à-dire à la concentration $(N / A)$ et non au nombre des spins contenus dans le volume de l'échantillon comme c'est le cas des méthodes usuelles de détection de la R. P. E. Cela signifie que dans les conditions $(N / A)=$ const. et $I=$ const. en variant la section du faisceau lumineux, le rapport signal-bruit reste constant. Les relations (15) et (17) montrent que pour $I=$ const. le nombre minimum de spins détectables $N_{\min }$ est proportionnel à l'aire $A$ illuminée de l'échantillon. Cette méthode est avantageuse dans le cas des échantillons petits sur lesquels sont concentrées des grandes intensités de lumière. Dans ce but on peut utiliser des faisceaux laser concentrés sur des aires $A$ très petites avec la condition qu'on n'atteigne pas la limite de détérioration de l'échantillon. Dans le cas des grands échantillons, on peut établir la distribution ponctuelle des centres paramagnétiques en déplaçant le faisceau laser.

3.2 En ce qui concerne la dépendance du nombre minimum de spins détectables $N_{\text {min }}$ par rapport à la fréquence R. P. E. $\left(\omega_{0}\right)$, nous observons que les sections transversales du D. C. M. et de la rotation Faraday sont proportionnelles à la polarisation électronique et celle-ci, dans le cas où sa valeur n'est pas trop grande, est proportionnelle à $\omega_{0}$. En conséquence, à $H_{1}^{2}=$ const., par les relations (15) et (17) nous obtenons le résultat important :

$$
N_{\min }=\frac{\text { const. }}{\omega_{0}}
$$

qui est en contraste avec le résultat correspondant dans le cas de la détection électromagnétique conventionnelle où nous avons $N_{\min }=$ const. $/ \omega_{0}^{15 / 4}[14]$ et à la détection bolométrique $N_{\min }=$ const. $/ \omega_{0}^{9 / 2}$ [15], c'est-à-dire une dépendance forte par rapport à la fréquence. Ce résultat montre que les avantages de la détection de la R. P. E. en utilisant l'effet Faraday par rapport à la détection conventionnelle ou bolométrique deviennent plus remarquables aux fréquences basses.

En tenant compte de ce que la composante imaginaire $\chi^{\prime \prime}$ de la susceptibilité dynamique est proportionnelle à $N \omega_{0}$, conformément à (18) nous arrivons à la conclusion que la détection de la R. P. E. en utilisant l'effet Faraday est caractérisé aussi par le fait que la susceptibilité dynamique minimale détectable $\chi_{\min }^{\prime \prime}$ est indépendante de la fréquence.

En ce qui concerne l'influence du facteur de saturation $s$, on peut dire que $N_{\min }$ est d'autant plus petit que le produit des temps de relaxation et du carré du champ magnétique de microondes est plus grand. Dans le cas $s=$ const., ce qui signifie un élargissement donné de la ligne par la saturation, nous remarquons une autre caractéristique importante de cette méthode, c'est-à-dire que $N_{\min }$ ne dépend pas des temps de 
relaxation ni du facteur de qualité de la cavité microondes.

3.3 Le nombre minimum des spins détectables dans le cas de la détection de la R. P. E. en utilisant l'effet Faraday est d'autant plus petit que sont plus grandes la sensibilité de la photocathode, l'intensité de la source de lumière, la section transversale efficace de l'effet Faraday et que la bande passante du système d'enregistrement est plus petite. Ainsi cette méthode est avantageuse dans le cas des échantillons ayant un grand produit de la section efficace d'absorption par le coefficient de dichroïsme circulaire magnétique.

On peut remarquer qu'à cause des relations de Kramers-Krönig, $\sigma_{\varepsilon}$ et $\sigma_{\varphi}$ ont des optima du même ordre de grandeur numérique pour une bande d'absorption donnée. C'est pour cela que d'après les relations (15) et (17) on peut dire que, dans les mêmes conditions, la sensibilité de la détection de la R. P. E. a le même ordre de grandeur soit en utilisant la rotation Faraday soit le D. C. M. L'utilisation de la rotation Faraday permet par contre des intensités de lumière plus grandes que l'utilisation du D. C. M., sans mettre en danger la vie du photomultiplicateur.
3.4 En ce qui concerne la longueur du chemin optique $l$, le rapport signal-bruit admet un maximum parce que l'effet Faraday est proportionnel à $l$ et que l'intensité de la lumière à la sortie de l'échantillon décroît exponentiellement avec $\gamma_{0} l$. Parce que l'intensité de la lumière se trouve à la puissance $\frac{1}{2}$ dans l'expression du rapport signal-bruit, il résulte que celui-ci devient maximum à $\gamma_{0} l=2$. Par exemple, dans le cas de l'utilisation du D. C. M., en remplaçant $I \operatorname{par} I \exp \left(-\dot{\gamma}_{0} l\right)$ dans la relation (16), nous trouvons pour $\gamma_{0} l=2$ :

$$
\frac{\delta i_{\mathrm{a} \text { eff }}}{\left(\overline{i_{f \mathrm{a}}^{2}}\right)^{1 / 2}}=\frac{2 \Gamma P s}{e(1+s)}\left(\frac{\eta I}{2 q \Delta f}\right)^{1 / 2} .
$$

La relation (19) est utile dans le sens qu'elle nous montre le rapport signal-bruit maximum qui peut être obtenu. Celui-ci ne dépend pas de la section efficace d'absorption $\sigma_{\mathrm{a}}$ ni de l'aire illuminée $A$ de l'échantillon ; le nombre de spins célibataires n'intervient pas directement. D'après la condition $\gamma_{0} l=2$ on obtient le nombre de spins de l'échantillon pour lequel le rapport signal-bruit prend la jvaleur maximum, à savoir $N=\left(2 \dot{A} / \sigma_{\mathrm{a}}\right)$.

\section{Bibliographie}

[1] Kastler, A., C. R. Hebd. Séan. Acad. Sci. 232 (1951) 953.

[2] OpechowsKi, W., Rev. Mod. Phys. 25 (1953) 264.

[3] Daniels, J. M., Wesemeyer, H., Can. J. Phys. 36 (1958) 405.

[4] Asawa, C. K., Satten, R. A., Phys. Rev. 127 (1962) 1542.

[5] Mollenauer, L. F., Pan, S., Yngvesson, S., Phys. Rev. Lett. 23 (1969) 683.

[6] Geschwind, S., Electron Paramagnetic Resonance, (Plenum Press) 1972, Ch. 5, 359-360.

[7] Margerie, J., Ann. Phys. 5 (1970) 15.
[8] Komarov, A. V., Riabtshenko, S. M., Prib. Tech. Exp. Nr. 4, 201 (1971).

[9] Panepucci, H., Mollenauer, L. F., Phys. Rev. 178 (1969) 589.

[10] Brown, F. C., Laramore, G., Appl. Opt. 6 (1967) 669.

[11] R. C. A. Technical Manual PT-60, 1963.

[12] Tătaru, E., St. Cerc. Fiz. 25 (1973) 901.

[13] Billardon, M., Ann. Phys. 7 (1962) 233.

[14] Poole, C. P., Electron Spin Resonance (Interscience Publishers, New York) 1967.

[15] Tătaru, E., Studia Univ. B. B. Physica, 18 (1973) fasc. $2,9$. 